\title{
Proposed procedure prompts more questions
}

\begin{abstract}
A ccording to the Public Health Service Policy on Humane Care and Use of Laboratory Animals ${ }^{1}$ and the Guide for the Care and Use of Laboratory Animals ${ }^{2}$ (Guide), it is the responsibility of the IACUC to understand federal and state regulatory requirements, distinguish pain and distress in animals from their normal state, to minimize or alleviate the pain and distress and to establish humane endpoints. Both the PHS Policy and the Guide expect procedures that may cause pain or distress to be performed with appropriate anesthesia and analgesia, unless justified for scientific reasons. At the same time, IACUC members also need to be aware that novel research procedures which haven't been fully characterized may cause pain and distress in some circumstances. In the proposed scenario at Great Eastern University, the attending veterinarian $(\mathrm{AV})$ only observed the videos of mice that have undergone procedure and suggests that animal should be placed in category $\mathrm{D}$ based on his observations and use of analgesics. The IACUC member, Collins, held a different point of view that a wire sticking out of the eye may not be painful, it might cause discomfort throughout the length of experiment, and therefore animals should be placed in category E. Both AV and the IACUC member have valid points, but they are more concerned about the pain category classification than ways to alleviate pain and distress and to establish humane end points.
\end{abstract}

The AV and Collins are classifying nonregulated species into USDA pain category for regulated species. Some IACUCs may choose to assign USDA pain categories to non-regulated species for monitoring reasons, but this is not required. As an IACUC member, we would like to know if the proposed procedure is part of a pilot study, the duration of experimentation after ocular implantations of device and if supplemental care will be provided to minimize discomfort, and whether there will be a need for post-procedural analgesia beyond the proposed timeline. Further queries include: duration and frequency of post-procedural monitoring for these animals; appropriate pain scoring criteria; humane end points; and intervention strategies to minimize or alleviate pain and distress for the proposed procedure. In addition, close monitoring of these animals will provide an insight on effectiveness of analgesics and supportive therapy or need for intervention strategies, including euthanasia to alleviate or minimize postprocedural distress or discomfort.

This scenario exemplifies the need for a post-approval monitoring (PAM) of the protocol by the AV and concerned IACUC member, including a report back to the IACUC with animal welfare observations. With PAM findings, the IACUC can reevaluate and request modifications to the protocol including, but not limited to, humane endpoints and intervention strategies as well as pain category classifications if necessary. Alternatively, the IACUC may approve a pilot study with a limited number of animals to better characterize the experimental procedures and establish appropriate humane end points.

In summary, we would recommend placing these animals in pain category $\mathrm{D}$ if Great Eastern University's IACUC policies require pain categories to non-regulated species, as the procedure is performed under anesthesia with adequate postprocedural analgesia with appropriate humane endpoints, thus alleviating the pain/distress.

Deepti Chadalavada ${ }^{1 *}$ and Sridhar Samineni ${ }^{2}$ ${ }^{1}$ NIAID/NIH (Charles River Contractor), Bethesda, MD, USA. ${ }^{2}$ Sobran-Inc, affiliated with Walter Reed Army Institute of Research, Silver Spring, MD, USA. *e-mail:deeptich9@gmail.com

Published online: 20 March 2019 https://doi.org/10.1038/s41684-019-0264-3

References

1. Institute for Laboratory Animal Research. Guide for the Care and Use of Laboratory Animals (National Academies Press, Washington, DC, 2001).

2. Public Health Service. Policy on Humane Care and Use of Laboratory Animals (US Department of Health and Human Services, Washington, DC, 1986; amended 2002).

\section{Disclaimer}

Material has been reviewed by the Walter Reed Army Institute of Research. There is no objection to its presentation and/or publication. The opinions or assertions contained herein are the private views of the author, and are not to be construed as official, or as reflecting true views of the Department of the Army or the Department of Defense.

\section{If there is any doubt, pilot it!}

W e agree with both Dr. Villanueva and Dr. Collins; therefore, if we were members of the Great Eastern University (GEU) IACUC, we would request an internal review of the situation.

On one hand, Dr. Villanueva is Great Eastern University's attending veterinarian (AV) and, thus, the institution's resident animal expert. His opinion should carry weight in this situation. The IACUC should give consideration to the fact that Dr. Villanueva has seen a visual presentation (via video) of the surgical procedure and ensuing care, which suggests that the procedure does not cause pain or distress in the animals. However, because he has not personally evaluated post-operative animals, he should be open to doing so.
Additionally, Dr. Collins' statement that, "a procedure that's painful or distressful to a human is likely to be painful or distressful to a mouse" shows that he has the right mind set with respect to animal welfare that is congruent with the regulatory stance on pain; however, does the same viewpoint apply to stress and distress? Many times, when we talk about alleviating stress/distress in the lab animal setting, we rely on animal acclimation and training. Do animals suffer the same level of stress as humans? Do they acclimate to stressful situations better than humans? Does acclimation really alleviate stress in animals? These are significant questions that may potentially be answered by an experimental study (i.e., a cortisol level study); however, we propose an easier way to address this micro-situation and bring peace to both Dr. Villanueva and Dr. Collins: a pilot study.

The IACUC could vote (as always, majority rules and votes are needed) to approve the project as a pilot study. A predetermined number of animals, perhaps 5 or 10 , will be subjected to the intravitreal injection procedure and closely monitored post-operatively for an appropriate period of time (e.g., 7 to 10 days). Ideally, Drs. Villanueva and Collins could monitor the animals personally, but in the spirit of cultivating a culture of trust, they may rely upon the principal investigator (PI), his/her staff, and the GEU animal care staff to monitor the animals' recovery. Since there is a doubt on the where the project 